\title{
Management of the stiff total knee arthroplasty with revision to a total condylar bearing rotating platform prosthesis
}

\begin{abstract}
Purpose: Evaluate the efficacy of revision knee surgery with rotating platform prosthesis for treating stiffness associated with total knee arthroplasty.

Methods: Between 2005 and 2007, twelve patients were treated by a single surgeon with a total condylar bearing rotating platform prosthesis for post-operative knee stiffness. Average age of patients was 60 years and the revision procedure was performed on average 8 months after the primary knee arthroplasty.

Results: Post-operatively, all patients had stability to varus/valgus and anterior/posterior testing, and all patients had normal overall alignment. Knee extension increased an average of 16.3 degrees, knee flexion increased an average of 30.1 degrees, and total range of motion increased an average of 46.4 degrees. Modified knee score utilizing the range of motion, alignment, and stability components of the Knee Society Clinical Rating System increased an average of 17.9 points. All improvements were statistically significant $(\mathrm{p}<0.05)$.

Conclusion: Revision to a total condylar bearing rotating platform prosthesis significantly improves ROM and function while maintaining stability and alignment for patient suffering from stiffness following a total knee arthroplasty.
\end{abstract}

Volume 6 Issue I - 2016

\author{
Sravan Dhulipala, John Fennessy, Timothy \\ Ganey, Jon Minter \\ Department of Orthopedics, Wellstar Atlanta Medical Center, \\ USA \\ Correspondence: Sravan Dhulipala, Department of \\ Orthopedics, Wellstar Atlanta Medical Center, Atlanta, USA, \\ Email sdhulip@gmail.com
}

Received: October 14, 2016 | Published: October 14, 2016

\section{Introduction}

Total knee arthroplasty (TKA) is performed to relieve pain and restore function in patients with arthritic knees. There are approximately 700,000 knee replacements performed each year, and this number is projected to reach 3.5 million per year by $2030 .{ }^{1}$ Although knee replacements offer significant improvements in quality of life, they are not without complications. Stiffness is a known complication that occurs in $1.3-12 \%{ }^{2-4}$ of primary total knee arthroplasties. Stiffness is defined as having a flexion contracture of 10-15 degrees or a total arc of motion that is less than 90 degrees. ${ }^{5,6}$

Several risk factors contribute to post-operative stiffness, including poor pre-operative ROM, delayed rehabilitation, infection, heterotrophic ossification, and revision surgery., It may be caused iatrogenically secondary to technical errors during surgery, such as using overly large components, over-tightening of the extensor mechanism, improper ligament balancing, or component malalignment. ${ }^{7-9}$

Treatment for stiffness involves physical therapy and manipulation under anesthesia to improve joint mobilization. ${ }^{2,5}$ Surgical treatments include arthroscopy with lysis of adhesions with or without posterior cruciate ligament excision, polyethylene exchange, and finally revision arthroplasty. ${ }^{8}$ Several prosthetic implants have been used in revision surgery, many of which employ a fixed bearing construct. However, there are no studies on revision total knee arthroplasty using mobile bearing constructs for stiffness. Mobile bearing prostheses have the theoretical advantage of having two articulating surfaces for the polyethylene insert and improved contact during full range of motion, which potentially reduces the stress on the polyethylene component, improves wear rate, and increases ROM.

Studies have shown that mobile bearing constructs have a survival rate of approximately $90 \%$ at twelve years, which is equal to or better than traditional fixed bearing constructs. ${ }^{10-12}$ The main risk with mobile bearing constructs is dislocation of the polyethylene insert, which only occurs in only $0.56 \%$ of mobile bearing implants used today. ${ }^{13}$

To our knowledge, there are no studies on the use of mobile bearing prostheses for stiffness in revision total knee arthroplasty. The purpose of our study is to evaluate the short and long term outcomes of stiffness in total knee arthroplasty that is treated with revision surgery to a mobile bearing prosthesis.

\section{Materials/Methods}

A retrospective chart review was performed from 2005 to 2007 to identify patients who underwent revision total knee arthroplasty for stiffness following a primary or revision total knee arthroplasty by the senior author. Stiffness was defined as a total arc of motion of 90 degrees or less or an extension lag of 20 degrees or more. Patients were excluded if they had an infection following their primary procedure. Eighteen patients met the inclusion criteria. Of these 18 patients, 12 had revision surgery with the Total Condylar 3 Rotating Platform prosthesis (Ace Depuy, Warshaw, Indiana), a mobile bearing prosthesis. All revision procedures were performed by a single surgeon.

Three primary variables were measured pre and post-operatively for each patient: alignment based on pre- and post-operative radiographs, stability to varus/valgus and anterior/posterior stress testing from physical examination, and range of motion on examination.

Using these variables, a modified knee score was calculated preand post-operatively from The Knee Society Clinical Rating System for all patients. Secondary to the retrospective nature of this study, the knees were only scored based on range of motion, stability, and alignment. Therefore, a knee with a full range of motion from $0-125$ degrees with medial, lateral, anterior, and posterior stability in normal alignment received a maximum score of 50 points. 


\section{Surgical technique}

A midline surgical approach was performed for the procedure. Full thickness medial and lateral skin flaps were mobilized. A medial arthrotomy was used to access the joint. No patella turndowns or quadriceps snips were performed. The extensor mechanism was mobilized by resecting the hypertrophic scar tissue in the infra-patellar area, supra-patellar pouch, medial gutter, lateral gutter and posterior capsule. After all components were resected, alignment and sizing was performed. After trialing the components, cement was used to secure the final components in place. All patients underwent continuous passive motion therapy and physical therapy postoperatively.

\section{Study demographics}

There were 5 males and 7 females in our study. The average age of the patients was 60 years at the time of the revision procedure with a range from 45 to 78 years. Osteoarthritis was the primary diagnosis for total knee replacement in 11 of the 12 patients, and rheumatoid arthritis was the primary diagnosis in the twelfth patient. The revision surgery was performed at an average of 16 months after the primary procedure (Range of 5-48 months).

All patients were diagnosed with post-operative stiffness using the definition described above. Eight of the twelve patients underwent at least one or more procedures to address their knee stiffness prior to our revision arthroplasty, including 7 manipulations under anesthesia (MUA), 2 arthroscopic lysis of adhesions, and 2 previous revisions with a fixed bearing construct.

\section{Results}

On clinical exam prior to revision surgery, patients had an average pre-operative range of motion from 20.5 +/- 13.7 degrees of extension to $71.8+/-21.1$ degrees of flexion. The average pre-operative arc of motion was $51.3+/-22.2$ degrees. Revision surgery was performed at an average of $8.0+/-1.4$ months after the index procedure and followup data was available at an average of $8.0+/-2.8$ months after the revision procedure. Patients had an average post-operative ROM from $4.2+/-5.0$ degrees of extension to $101.9+/-18.5$ degrees of flexion. The average post-operative arc of motion was $97.8+/-21.7$ degrees. Post-operative flexion increased an average of $30.1+/-25.2$ degrees, extension increased an average of $16.3+/-11.2$ degrees, and total arc of motion increased as average of $46.4+/-24.6$ degrees. All changes in ROM were statistically significant $(\mathrm{p}<0.05)$. Table 1 summarizes these results and Figure 1 illustrates the changes in flexion, extension, and total arc of motion. Eleven of the twelve patients had an improvement in extension and eleven of twelve had an improvement in flexion. All patients had an increase in total arc of motion.

Table I Summary of range of motion

\begin{tabular}{lll}
\hline & Average & P-value \\
\hline Pre-op extension & $20.5+/-13.7$ & \\
Pre-op flexion & $71.8+/-21.1$ & \\
Pre-op ROM & $51.3+/-22.2$ & \\
Pre-op knee score & $14.4+/-4.8$ & \\
Post-op extension & $4.2+/-5.0$ & \\
Post-op flexion & $101.9+/-18.5$ & \\
Post-op ROM & $97.8+/-21.7$ & \\
Post-op knee score & $32.1+/-11.7$ & \\
Change extension & $16.3+/-11.2$ & $3.78 \times 10^{-4}$ \\
Change flexion & $30.1+/-25.2$ & $1.64 \times 10^{-3}$ \\
Change ROM & $46.4+/-24.6$ & $4.25 \times 10^{-5}$ \\
Change knee score & $17.8+/-12.4$ & $4.36 \times 10^{-4}$ \\
\hline
\end{tabular}

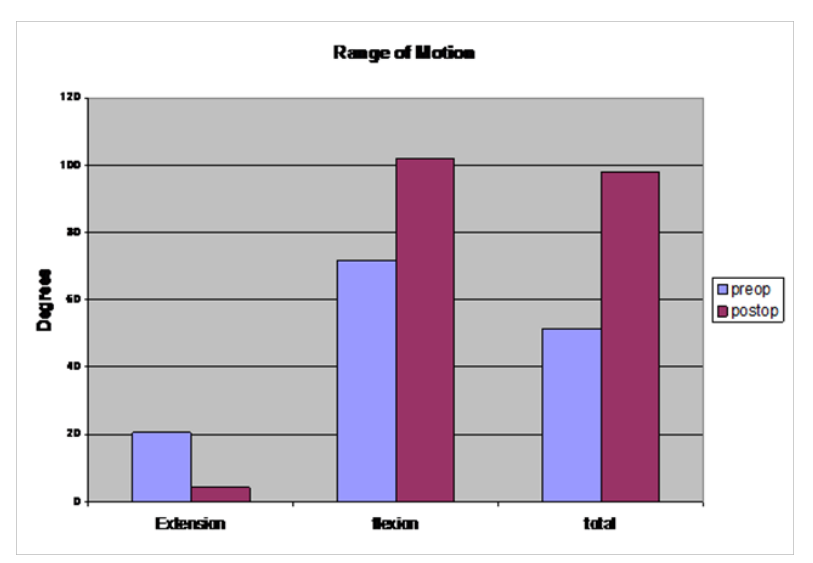

Figure I Illustration of improvements in mean extension, flexion, and total arc of motion.

Pre-operatively, two patients had mild varus alignment and one had mild valgus alignment on radiograph. On examination, one patient had varus/valgus instability with stress testing. Post-operatively, all patients had normal overall alignment of the prosthesis on radiographs, and all patients were stable to varus/valgus and anterior/ posterior stress testing.

Using the modified knee score from the Knee Society Clinical Rating System described above, the average pre-op knee score was $14.3+/-4.8$ out of 50 points. The post-operative knee score increased to $32.1+/-11.7$ out of 50 points. There was an average increase of 17.8 $+/-12.4$ points, which was statistically significant $(\mathrm{p}<0.05)$. (Table 2) Four of the twelve patients complained of pain post-operatively that affected their functional ability. However, ten of twelve patients stated that overall they were satisfied with their revision procedure. One patient, who was not satisfied, had a loss of 10 degrees of flexion with a 1 degree improvement in extension; this patient had an overall increase in arc of motion of only 4 degrees and had an increase in the modified knee score of only 1 point. The second patient who stated that they were not satisfied with the revision had an improvement in flexion of only2 degrees but had an improvement in extension of 43 degrees. His modified knee score increased by 9 points.

Table 2 Modified knee score based on range of motion, alignment, and stability of patients pre- and post-op

\begin{tabular}{|c|c|c|c|c|}
\hline Patient & Pre-op Score & Post-op Score & Change & Patient Satisfied \\
\hline I & II & 20 & 9 & No \\
\hline 2 & 15 & 16 & I & No \\
\hline 3 & II & 21 & 10 & Yes \\
\hline 4 & 14 & 23 & 9 & Yes \\
\hline 5 & 5 & 46 & 41 & yes \\
\hline 6 & 19 & 30 & II & yes \\
\hline 7 & 12 & 46 & 34 & yes \\
\hline 8 & 10 & 34 & 24 & yes \\
\hline 9 & 19 & 24 & 5 & yes \\
\hline 10 & 22 & 50 & 28 & yes \\
\hline II & 19 & 43 & 24 & yes \\
\hline 12 & 15 & 32 & 17 & yes \\
\hline Mean & 14 & 32 & 17.8 & $10 / 12$ \\
\hline
\end{tabular}

Post-operative complications were present in four patients. Two patients required a MUA after the revision procedure. Two other patients suffered from neuropathy in the operative leg following the revision. There were no post-operative infections in the patient population. 


\section{Discussion}

The purpose of our study was to determine the efficacy of revision arthroplasty with a mobile bearing prosthesis for stiffness following a total knee replacement. Our study included 12 patients who all achieved improvements in total ROM following surgery.

Treatment of stiffness should begin with the least invasive procedure and progress to more invasive interventions if needed. Manipulation under anesthesia is the primary procedure that is performed. Keating et al. reported a $90 \%$ success rate with MUA with an increase of approximately 35 degrees in total arc or motion at five years follow-up. ${ }^{14}$ More invasive interventions include arthroscopic lysis of adhesions with or without PCL excision, polyethylene insert exchange with open arthrolysis, and revision arthroplasty. Babis et al. ${ }^{15}$ attempted a tibial polyethylene exchange to a thinner insert along with arthrolysis of adhesions to improve ROM in stiff knees. They showed only a modest improvement of 19.4 degrees in total ROM in five patients with a high rate of complications including two post-operative infections and four patients with severe pain. All five patients continued to complain of stiffness in their study. They concluded that polyethylene exchange should be reserved as a last resort for treatment of stiffness..$^{15}$ Mont et al. looked at improving ROM through a combination of open arthrolysis and intensive post-operative rehabilitation. They found an average increase of 31 degrees in ROM in $94 \%$ of the patients in their study. ${ }^{16}$ Williams et al. showed good results with arthroscopic release of the PCL in stiff knees. Eighty-eight percent of patients in their study expressed satisfaction with the surgery. They showed an increase in flexion from 73.9 degrees pre-op to 104.5 degrees (increase of 30.6 degrees) at 20 month follow-up. Extension only improved form 4 degrees pre-op to 1.3 degrees at follow-up. Thus, arthroscopic PCL excision should be utilized in patients with a PCL retaining prosthesis with limitations primarily in flexion. ${ }^{17}$

Revision total knee arthroplasty is often a last resort for treatment of stiffness. It has shown good results in most patients. The earliest report of revision total knee arthroplasty for stiff total knees was presented by Nicholls et al in 1990. In this series, patients had an increase in range of motion of 32 degrees. Eight of twelve revisions were performed for malpositioned components and eleven of twelve patients showed improvement in pain. Revision procedures were performed with various implant types. ${ }^{18} \mathrm{Kim}$ et al reported the largest series of knee revisions with 56 patients. They found that poor preoperative flexion and extension was a significant risk factor for postoperative stiffness. They found an improvement of mean flexion from 65.8 to 85.4 degrees, improvement of mean arc of motion from 54.6 to 82.2 degrees, and improvement of extension from 11.3 to 3.2 degrees. ${ }^{19}$ Similarly, Haidukewych et al..$^{20}$ reported a 33 degree increase in ROM, but only a $67 \%$ patient satisfaction amongst their fifteen patients with $25 \%$ requiring additional interventions for residual stiffness. ${ }^{20}$ The best results from revision total knee arthroplasty were reported by Christensen at all in 2002. They reported a $100 \%$ patient satisfaction with a mean improvement in ROM of 43.5 degrees and mean improvement in extension of 12.3 degrees. Patients in this study were revised with posterior stabilized condylar prosthesis. ${ }^{21}$

Table 3 illustrates a summary of the various studies utilizing revision arthroplasty for stiff total knees. Our study is the only known study using rotating platform prosthesis. Our data shows similar results to those reported by Christensen in regards to improvements in ROM (46.2 versus 43.5 degrees). Our patient satisfaction is lower than their reported $100 \%$. Factors related to this include residual pain and stiffness. Of the three patients that had a post-operative ROM less than 90 degrees, two patients were not satisfied with results of the revision surgery. All patients in our study who had ROM greater than 90 degrees were satisfied.

Table 3 Clinical results for various studies utilizing revision Arthroplasty for stiff total knee replacements ${ }^{18-21}$

\begin{tabular}{|c|c|c|c|c|c|}
\hline Study Author & $\mathbf{N}$ & Mean f/u & $\begin{array}{l}\text { Implant } \\
\text { Used }\end{array}$ & $\begin{array}{l}\text { Change } \\
\text { ROM }\end{array}$ & $\begin{array}{l}\text { Patient } \\
\text { Satisfaction }\end{array}$ \\
\hline Nicholls ${ }^{18}$ & 13 & $\begin{array}{l}\text { Mean } \\
\text { unreported } \\
\text { (2-7 year } \\
\text { range) }\end{array}$ & Mixed & 32.2 & $92 \%$ \\
\hline Christenson $^{21}$ & 11 & 37.6 & $\begin{array}{l}\text { Posterior } \\
\text { Stabilized }\end{array}$ & 43.5 & $100 \%$ \\
\hline Haidukewyche ${ }^{20}$ & 16 & 42 & $\begin{array}{l}\text { Posterior } \\
\text { stabilized }\end{array}$ & 33 & $66 \%$ \\
\hline $\mathrm{Kim}^{19}$ & 56 & 43 & $\begin{array}{l}\text { Posterior } \\
\text { stabilized }\end{array}$ & 27.6 & Unreported \\
\hline Minter & 12 & 8 & $\begin{array}{l}\text { Rotating } \\
\text { Platform }\end{array}$ & 46.4 & $83 \%$ \\
\hline
\end{tabular}

An obvious shortcoming in our study is the short follow-up of patients (average 8 months). Only 3 of 12 patients had data available 1 year or more after the revision procedure. We did attempt to contact our patients approximately 10 years following the revision procedure, but eleven were lost to follow-up secondary to death, moving away, or invalid contact information. Additionally, as our study was retrospective, we were not able to do full calculations of the Knee Society Clinical Rating System score and were limited to scoring only the range of motion, alignment, and stability portions of the score. Thus we were unable to compare our knee scores with those reported in other studies. Our sample size of 12 patients is small, but comparable to most other studies on revision arthroplasty for stiff knees.

\section{Conclusion}

Revision total knee arthroplasty remains a viable option for patients with stiff total knees after failing conservative treatment. Our results utilizing rotating platform prosthesis showed improvements in ROM comparable to and slightly better than that reported in other studies. However, a randomized prospective clinical trial would be needed with sufficient power to clearly justify use of one prosthesis over another.

\section{Acknowledgments}

None.

\section{Conflicts of interest}

None.

\section{References}

1. Kurtz S, Ong K, Lau E, et al. Projections of primary and revision hip and knee arthroplasty in the United States from 2005 to 2030. J Bone Joint Surg Am. 2007;89(4):780-785.

2. Yercan HS, Sugun TS, Bussiere C, et al. Stiffness after total knee arhtroplasty: prevalence, management and outcomes. Knee. 2006;13(2):111-117.

3. Gandhi R, de Beer J, Leone J, et al. Predictive risk factors for stiff knees in total knee arthroplasty. J Arthroplasty. 200621(1):46-52.

4. Nelson CL, Kim J, Lotke PA. Stiffness after total knee arthroplasty. $J$ Bone Joint Surg Am. 2005;87Suppl1(Pt 2):264-270.

5. Maloney WJ. The stiff total knee arthroplasty: evaluation and management. $J$ of Arthroplasty. 2002;17(4 Suppl 1):71-73. 
6. Panni AS, Cerciello S, Vasso M, et al. Stiffness in total knee arthroplasty. J Orthop Traumatol. 2009;10(3):111-118.

7. Dennis DA. The stiff total knee arthroplasty: causes and cures. Orthopedics. 2001;24(9):901-902.

8. Scuderi GR. The stiff total knee arthroplasty: causality and solution. $J$ Arthroplasty. 2005;20(4 Suppl 2):23-26.

9. Kelly MA, Clarke HD. Stiffness and ankylosis in primary total knee arthroplasty. Clinical Orthop \& Related Research. 2003;416:68-73.

10. Papachristou G, Plessas S, Sourlas J, et al. Cementless LCS rotating platform knee arthroplasty in patients over 60 years without patella replacement: a mid-term clinical outcome study. Med Sci Monit. 2006;12(6):264-268.

11. Ali MS, Mangaleshkar SR. Uncemented rotating-platform total knee arthroplasty: a 4-year to 12-year follow-up. J Arthroplasty. 2006;21(1):80-84.

12. Sorrells RB, Voorhorst PE, Murphy JA, et al. Uncemented rotatingplatform total knee replacement: a five to twelve-year follow-up study. J Bone Joint Surg Am. 2004;86(10):2156-2162.

13. Fisher DA, Bernasek TL, Puri RD, et al. Rotating platform spinouts with cruciate-retaining mobile-bearing knees. $J$ Arthroplasty. 2011;26(6):877-882.
14. Keating EM, Ritter MA, Harty LD, et al. Manipulation after total knee arthroplasty. J Bone Joint Surg Am. 2007;89(2):282-286.

15. Babis GC, Trousdale RT, Pagnano MW, et al. Poor outcomes of isolated tibial insert exchange and arthrolysis for the management of stiffness following total knee arthroplasty. J Bone Joint Surg Am. 2001;83(10):1534-1536.

16. Mont MA, Seyler TM, Marulanda GA, et al. Surgical treatment and customized rehabilitation for stiff knee arthroplasties. Clin Orthop Relat Res. 2006;446:193-200.

17. Williams RJ, Westrich GH, Siegel J, et al. Arthroscopic release of the posterior cruciate ligament for stiff total knee arthroplasty. Clin Orthop Relat Res. 1996;331:185-191.

18. Nicholls DW, Dorr LD. Revision surgery for stiff total knee arthroplasty. Journal Arthroplasty. 1990;5:S73-S77.

19. Kim J, Nelson CL, Lotke PA Stiffness after total knee arthroplasty. J Bone Joint Surg Am. 200486(7):1479-1484.

20. Haidukewych GJ, Jacofsky DJ, Pagnano MW, et al. Functional results after revision of well-fixed components for stiffness after primary total knee arthroplasty. J Arthroplasty. 2005;20(2):133-138.

21. Christensen CP, Crawford JJ, Olin MD, et al. Revision of the stiff total knee arthroplasty. J Arthroplasty. 2002;17(4):409-415. 